\title{
Bending improvement in Spot Heating of pipes in comparison with Line Heating method
}

\author{
Seyed Ehsan Chavoshi ${ }^{1}$ and Seyed Ebrahim Moussavi Torshizi ${ }^{2, *}$ \\ ${ }^{1}$ Department of Mechanical and Energy Engineering, Shahid Beheshti University, Tehran, Iran \\ ${ }^{2}$ Faculty of Mechanical and Energy Engineering, Shahid Beheshti University, Tehran, Iran
}

Received: 24 May 2018 / Accepted: 3 April 2019

\begin{abstract}
In Spot Heating, a small area of a metal part surface is heated quickly with a gas torch, laser beam, or induction coil to a temperature below the phase change temperature and then cools down. The heated area undergoes compressive plastic strain and the part gets deformed. This method is usually applied as trial and error for straightening shafts, bridge components, ship structures, etc. The conventional straightening mechanism in industries involves creating thermal gradient mechanism (TGM) and shortening. Many studies have been conducted for bending of thin pipes (at a maximum thickness of $2 \mathrm{~mm}$ ) with the induction of "shortening" by laser. Spot Heating, despite its simplicity, results in very small deformations. The present study aims to increase the deformation in the Spot Heating method so as to extend its use in pipe straightening. To meet this goal, the shortening mechanism is developed through a thick pipe wall by optimizing the heating parameters. CFD analysis of flame flow is carried out to determine the heat flux distribution over the pipe surface. Also, the finite element method and optimization are used to analyze and raise the pipe deformation mechanism. The results indicate a considerable increase in the pipe bending which reduces the stages necessary for the pipe straightening in industries. Furthermore, the appropriate distance for combining the hot spots is also obtained. To evaluate the results, the Spot Heating test is performed, showing appropriate agreement with the simulation results.
\end{abstract}

Keywords: Spot Heating / pipe straightening / flame bending / finite element method

\section{Introduction}

Shape correction of pipes, shafts, and bridges is a practical application in various industries. Generally, shafts of rotating equipment, such as turbines and compressors, are distorted during the operation and, consequently, require straightening. Also, during the execution of pipelines, the precise adjustment of pipes at the junction faces problems and requires straightening. For straightening shafts, various methods such as cold and hot bending, blasting, heat treatment, Spot Heating, etc. are developed.

In a Spot Heating, a small area of a metal part is heated by a gas torch, laser beam, induction coil, etc. in a few seconds. To avoid contact with gas, the surrounding areas are covered with insulation layers. The heated zone expands, softens, and undergoes plastic strain under the pressure of the surrounding cold and stiff zone. This leads to a slight bulging, reducing the length of the heated zone and deformation of the part after cooling. Using a gas torch, the method becomes simple and inexpensive.

\footnotetext{
* e-mail: e moussavi@sbu.ac.ir
}

One of the limitations of Spot Heating is the very low deformations and the impossibility of reheating a zone to increase displacements $[1,2]$. Although it is possible to apply compressive prestress in the heating zone to increase bending angle $[3,4]$, this requires a suitable tool for applying preload to the piece and is restricted to the buckling strength and yield limit of the part. One of the important issues in the Spot Heating of pipes is the possibility of inducing tensile residual stresses equal to the yield stress. Addition of working stresses to this residual stress may result in re-yielding and reducing fatigue life [5].

"Line Heating" is a robust method for increasing the bending in pipe straightening where the heat source is moved along certain paths. This method requires the identification of suitable paths and control of the torch movement. The use of Line Heating along longitudinal reciprocating paths in pipes (Holt method - Fig. 1) is an old method for straightening pipes. This method leads to a significant increase in the bending compared to Spot Heating. Moreover, it requires the two torches to move with the same speed on both sides of the pipe, making its implementation complicated $[5,6]$. 


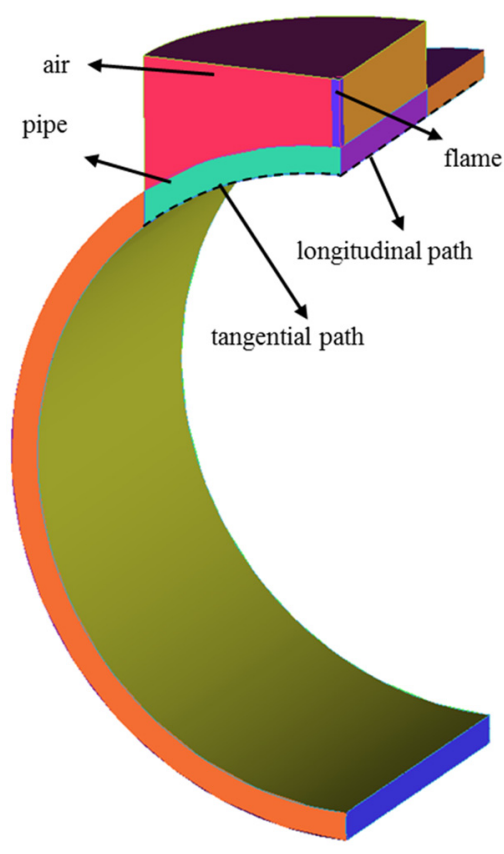

Fig. 1. Fluid and solid domain.

Gatto et al. [5] conducted some tests to investigate the straightening of pipes by Spot Heating and Holt's method. They applied Spot Heating by a torch in a small area (about $20 \mathrm{~mm}$ radius) of pipes of $150 \mathrm{~mm}$ diameter and $12.5 \mathrm{~mm}$ thickness. So, bending angles of the order of $100 \mathrm{~mm} / \mathrm{m}$ of pipe length were created. Using the Holt method, they also increased the displacements to $2.5 \mathrm{~mm} / \mathrm{m}$ in pipes with a diameter of $200 \mathrm{~mm}$ and a thickness of $7.9 \mathrm{~mm}$.

The Finite Element Method (FEM) is applied for simulation of thin-walled pipes using laser [7-9]. The simulation of plate forming by Line Heating was carried out in numerous references [10-12]. Poursaeidi et al. [2,13] performed the simulation by applying a local temperature change in a power plant shaft.

In the present study, Spot Heating parameters are optimized to increase pipe deformation. To achieve this goal, the shortening mechanism is developed through a thick pipe wall by optimizing the heating parameters. Computational Fluid Dynamics (CFD) analysis of flame flow is carried out to determine the heat flux distribution over the pipe surface and compare it with Gaussian distribution. Simulation by FE method and optimization are used to analyze and increase the deformation mechanism. Moreover, the appropriate distance for combining hot spots to increase the deformation is also determined. To evaluate the results, the Spot Heating test is performed.

\section{CFD analysis}

The initial optimization results (represented in Sect. 4) indicated that the optimum radius of the heating zone is largely relative to the pipe diameter. Therefore, heat distribution is expected to be significantly different from

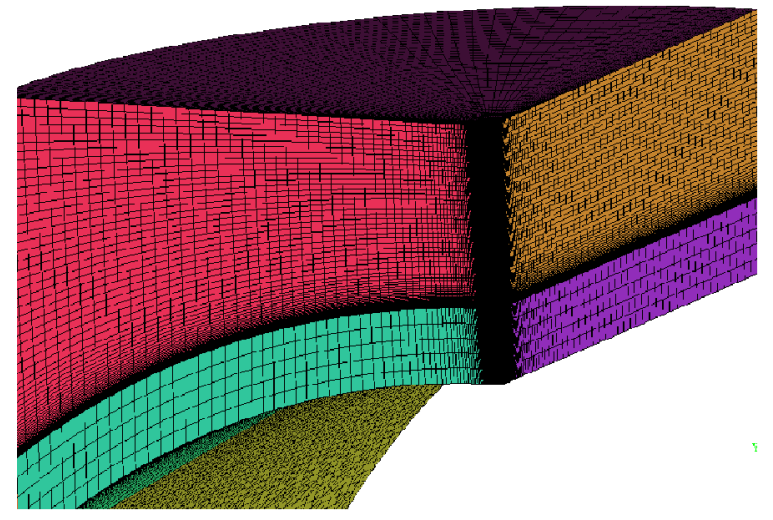

Fig. 2. Meshing of the computational domain in CFD analysis.

Gaussian distribution. So, for the sake of accuracy, the torch was modeled as an impinging jet.

Some studies applied a predetermined temperature $[12,14]$. Shahidi et al. modeled the torch as an impinging jet in the Line Heating [15]. Gaussian distribution of heat flux is used for its simplicity in multiple studies $[11,16]$.

Woo and Shin [17] studied the heat transfer between a plate and a torch using a simplified axisymmetric CFD model to analyze the velocity distribution of the flow. Then, using experimental relations for Nusselt number, the coefficient of convection and the heat flux were obtained. They fitted a Gaussian distribution to their results to make a comparison and showed that the temperature difference between the Gaussian distribution and that of their research increased over time. This was due to the fact that the heat transfer between the flow and the plate decreased with increasing plate temperature, but this was not the case in the Gaussian distribution.

To determine the heat flux applied to the pipe surface, ANSYS Fluent was used to conduct CFD analysis of flame flow and thermal analysis of pipe (Conjugate Heat Transfer). The surroundings of the torch were modeled as fluid environment and the pipe was modeled as a solid (Fig. 1). The domain was meshed using ANSYS ICEM CFD software, taking into account the boundary layer conditions (Fig. 2). The pipe surface was defined as the coupling interface between the solid and fluid domains. Fluid analysis was considered two-phase: air and premixed flame. The $k-e$ model was used to apply the turbulence effect, and the temperature of the oxyacetylene flame in a neutral state was $3000^{\circ} \mathrm{C}$ (according to the study conducted by Woo and Shin [17]). Determining the temperature distribution of different flames at the nozzle outlet requires the simulation of the combustion process. However, in this study, the flame temperature at the torch outlet is assumed to be constant and the results of torch calibration test were incorporated to correct the resulting error. Therefore, flame conditions including discharge, pressure, average temperature, and distance from the plate surface are adjusted so that the temperature variation matches the calibration test results.

Figure 3 shows the heat flux distribution obtained from the CFD analysis at different times. As can be seen, heat 


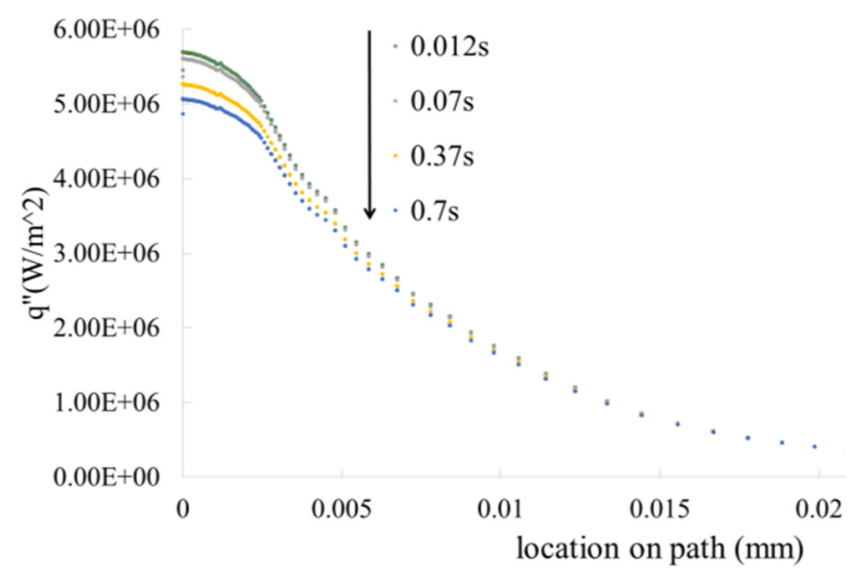

Fig. 3. Heat flux distribution along the tangential path at different times.

flux changes over time which is not addressed in the Gaussian distribution. Also, the velocity distribution is shown in Figure 4 where a stagnation point is created at the jet impingement location, leading to a reduction in its heat flux. This issue is not included in the Gaussian distribution.

Figure 5 shows the difference between heat flux distribution across the tangential and longitudinal paths.

As a conclusion, three factors induce the difference between Gaussian and real heat flux distribution, namely temperature changes in pipe surface over time, stagnation point of flame, and pipe shape.

\section{FE simulation of Spot Heating}

\subsection{Simulation settings}

The presence of two field variables, namely displacement and temperature, causes the coupling of structural and thermal equations through the thermal expansion coefficient and temperature-dependency of material properties. ANSYS software was used to simulate the process by the FE method. This software is capable of direct and sequential modeling of coupled thermoelastic and thermoplastic problems. The heat produced by plastic work was very low and can be ignored. Furthermore, the heating-induced pipe deformation was negligible and does not affect the thermal loads. Therefore, it was possible to solve the coupled thermal-structural problem sequentially (aka load vector method). Thus, thermal analysis was first performed and then the solution results, including temperature distribution, were transferred to the structural analysis as load vector.

Considering that the fixed end of the pipe was far from the heating zone in the tests, a quarter model of the pipe was modeled due to the pipe symmetry. The outer diameter of the pipe is $219.56 \mathrm{~mm}$, and its thickness is $8.18 \mathrm{~mm}$. The pipe loading and boundary conditions are shown in Figure 6. In the first analysis step, thermal flux was applied until the maximum surface temperature of $700^{\circ} \mathrm{C}$ (below metallurgical phase change temperature of carbon steels) and then removed in the second step.

CFD analysis of flame flow, represented in Section 2, showed the differences between Gaussian distribution and
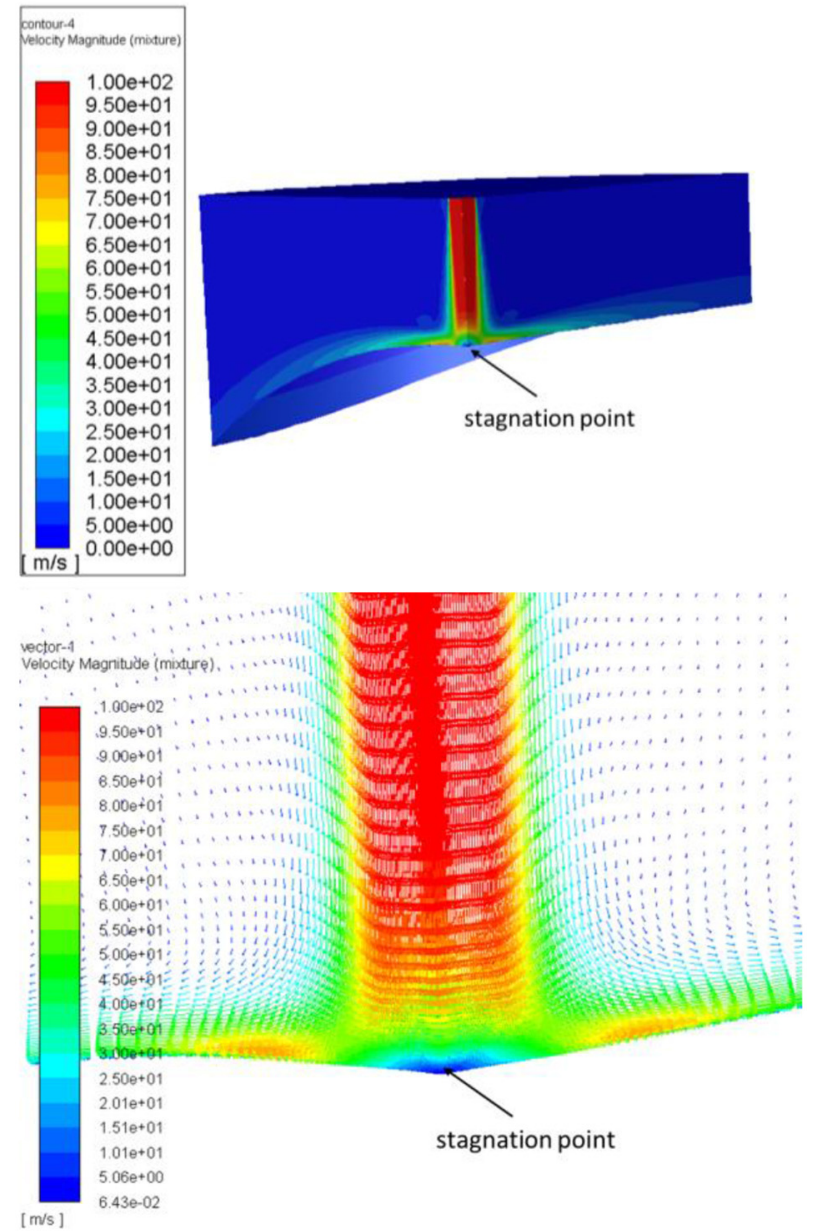

Fig. 4. Velocity distribution in the fluid domain.

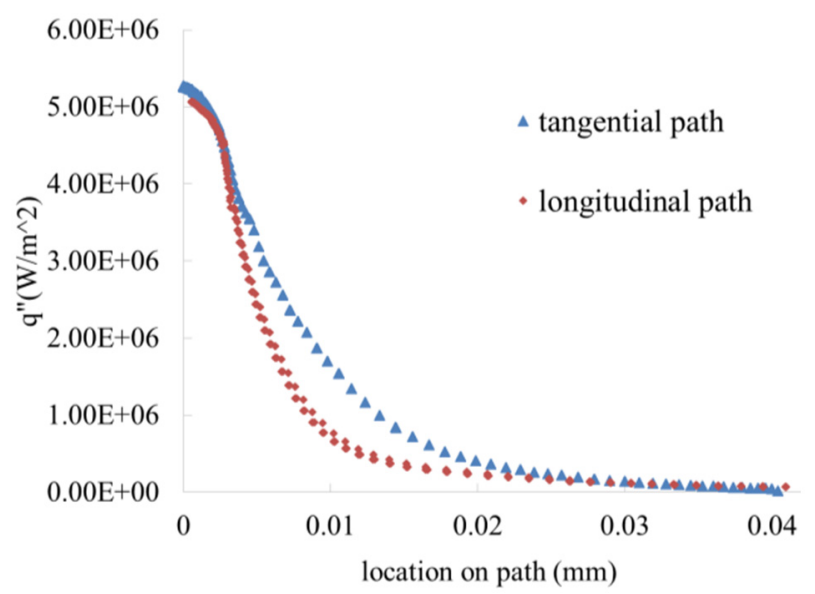

Fig. 5. Comparison of heat flux between tangential and longitudinal paths.

real distribution of heat flux. However, using the torch calibration test, the Gaussian distribution parameters can be adjusted such that the temperature variations in the finite element model (FEM) become consistent with the test results. FEM analyses revealed that the Gaussian 


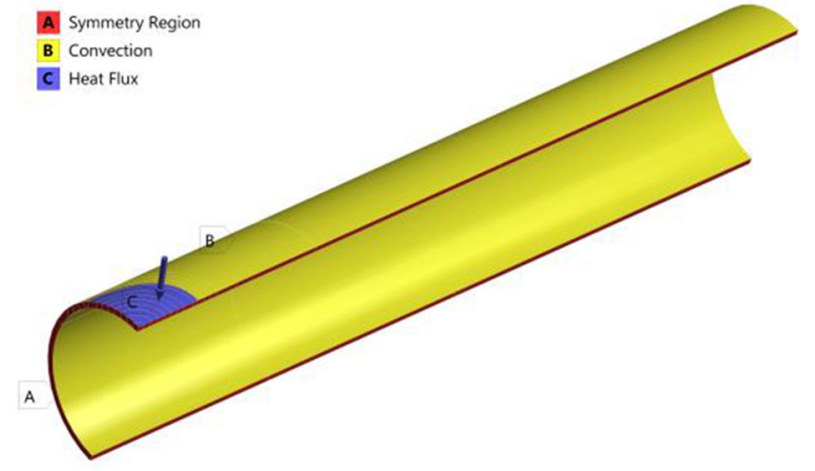

Fig. 6. Geometry and thermal loading of the pipe quarter model.

distribution assumption yielded negligible error (under 5\%) on the final pipe bending. Thus, the heat flux was applied as a Gaussian distribution according to the equation $q^{\prime \prime}(r)=q_{\max }^{\prime \prime} e^{-\gamma r^{2}}$, where $q_{\max }^{\prime \prime}$ is the maximum heat flux, $\gamma$ stands for the concentration coefficient, and $q^{\prime \prime}(r)$ is the heat intensity at any given radius $(r)$. The maximum heat flux value of $2.5 \mathrm{~W} / \mathrm{mm}^{2}$ and the effective radius of $20 \mathrm{~mm}$ were set as the initial values of the present study.

The effect of surrounding air was modeled as natural convection (Newton's law). The convection coefficient was incorporated based on the natural convection coefficients of a horizontal cylinder. Nevertheless, the FE analyses showed that the results were not sensitive to the exact values of convection coefficients. Symmetry faces of the pipe were thermally insulated. In structural analysis, the normal displacement of the symmetry faces was constrained. Also, to prevent rigid body motion, the displacement of the lower point (at the intersection of two faces of symmetry in the heating direction) was constrained. The full Newton-Raphson solver was used due to the nonlinearity of the analysis. Analyses showed that radiation has no significant effect on the results.

\subsection{Material properties}

The pipe material is SA-106 (grade C) carbon steel with $0.192 \%$ carbon observed by the Quantometer test. The average yield stress of $295 \mathrm{MPa}$ and tangent modulus of $4900 \mathrm{MPa}$ were obtained from the tension test. Varma and Sohn [4] described general relations for the modulus of elasticity, tangent modulus, and yield stress of low-carbon steels in terms of temperature. So, the mechanical properties were obtained from both the relations and tension test results. The values of the density, coefficient of thermal expansion, specific heat capacity, etc. were derived from Clausen study [10]. In addition, the material was assumed to be homogeneous. Von Mises yield criterion and kinematic hardening model were applied due to low plastic strains.

\subsection{Meshing}

Solid90 and Solid186 second-order elements were used in ANSYS software for thermal and structural analysis, respectively. These elements are hexahedral and isoparametric [18].

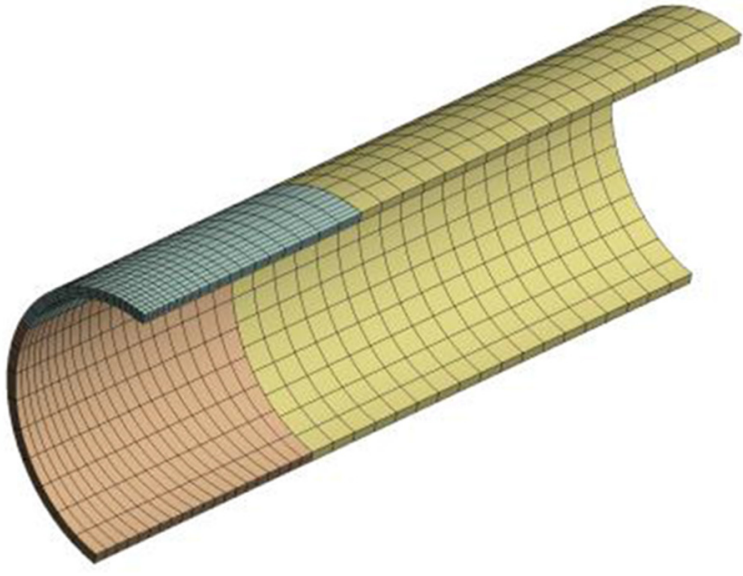

Fig. 7. Meshing of the pipe quarter model.

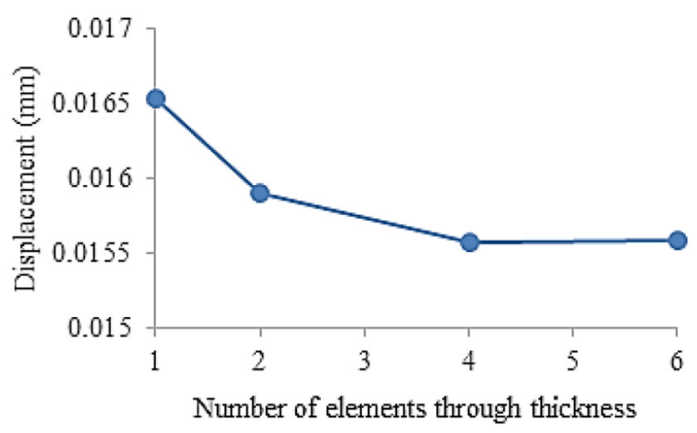

Fig. 8. Variation of the final displacement vs. number of elements through the thickness.

The size of the elements used in the pipe model should be so small so that the gradient of quantities in different directions (especially through thickness) gets properly captured. A view of the pipe quarter model mesh is shown in Figure 7.

The final displacement of the pipe was the result of plastic strains and stresses generated in the heating and cooling stages. Therefore, to determine the independence of the analytical results from the mesh size, variations in the final bending of pipe were investigated against the elements size for a pipe of $550 \mathrm{~mm}$ length (Fig. 8). As can be seen, it is enough to use four second-order elements along the pipe thickness. Considering the minimum heating zone diameter, the size of $10 \mathrm{~mm}$ for the surface elements is sufficient.

\section{FE simulation results}

\subsection{Pipe bending optimization}

A parametric study was conducted to increase the tube deformation by determining the relationship between the amount of pipe bending per unit length of pipe after cooling $(\delta)$ and flame parameters. The flame parameters include the maximum heat intensity $q_{\max }^{\prime \prime}$ and the effective radius $R_{e}$ (the radius where $q^{\prime \prime}\left(R_{e}\right)=(1 / e) q_{\max }^{\prime \prime}$ which leads to the relation $\left.R_{e}^{2}=1 / \gamma\right)$. The heating time was introduced into 


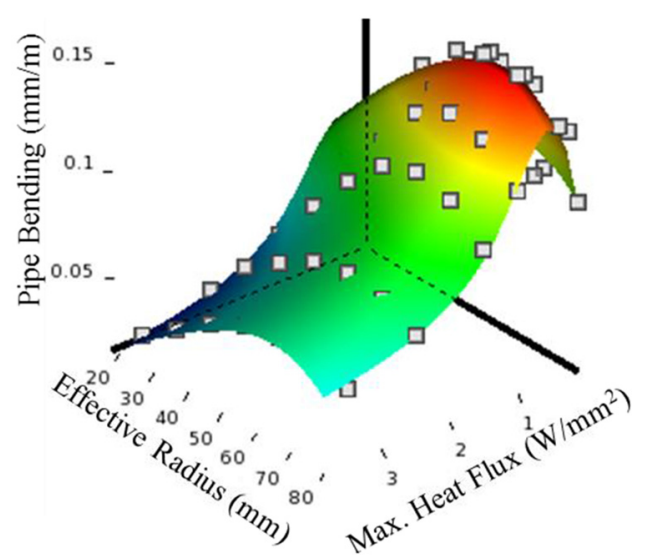

Fig. 9. Pipe bending $\delta$ vs. effective radius $R_{e}$ and maximum heat flux $q_{\max }^{\prime \prime}$.

the problem in the form of surface maximum temperature. To do this, the heating time was controlled to a temperature of up to $700^{\circ} \mathrm{C}$, which made it easier to interpret the results. In order to achieve the maximum bending of the pipe, the optimization process was performed using the "response surface" method. In this method, the "design of the experiments" was first done based on the problem parameters. Then, the determined design points (various combinations of parameters) were solved and the response surface was obtained. In this study, the "Genetic Aggregation" algorithm was employed for determining the response surface. Finally, the optimal point was obtained by screening the response surface. Figure 9 depicts the pipe bending changes in terms of $R_{e}$ and $q_{\max }^{\prime \prime}$. The maximum bending is $0.149 \mathrm{~mm} / \mathrm{m}$ at an effective radius of $65 \mathrm{~mm}$ and an intensity of $0.5 \mathrm{~W} / \mathrm{mm}^{2}$. This value is more than 3 times the displacement of conventional local heating (including the study conducted by Gatto et al. [5]).

\subsection{A discussion on the pipe deformation}

Different studies have discussed the deformation of thin pipes (up to $2 \mathrm{~mm}$ ) by laser heating. Three deformation mechanisms have been proposed in different studies, namely, thermal gradient mechanisms (TGMs), shortening (aka upsetting), and local buckling $[19,20]$. TGM occurs in straightening shafts by Spot Heating [5,21]. In the laser bending of thin pipes (up to $2 \mathrm{~mm}$ ), shortening mechanism takes place $[19,20]$. Thicker pipes have different deformation mechanisms under the Gaussian distribution of heat flux.

The temperature increase of the pipe through the thickness depends on $R_{e}, q_{\max }^{\prime \prime}$ and duration of the heating. High values of $q_{\max }^{\prime \prime}$ and low values of $R_{e}$ (relative to thickness) result in a rapid increase in temperature. The pipe thickness is not completely heated before reaching the maximum temperature. Thus, a small portion undergoes plastic strain and bends locally. This type of heating results in a temperature gradient mechanism where the pipe bending is very small. As shown in Figure 9, low values of

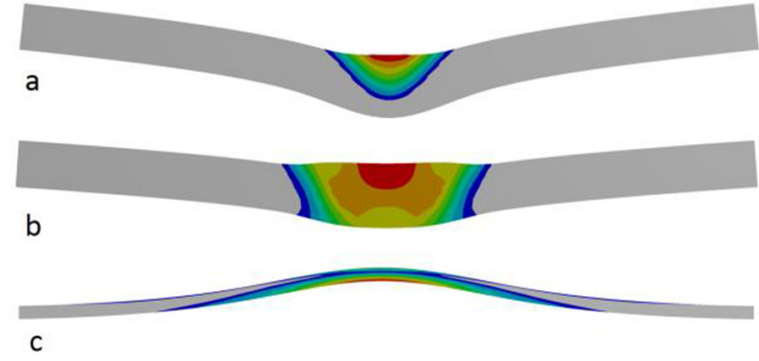

Fig. 10. Deformation mechanisms and plastic strain in Spot Heating (a) thermal gradient, (b) shortening, (c) local buckling.

$R_{e}$ and high values of $q_{\max }^{\prime \prime}$ lead to small values of $\delta$. Figure 10a shows the deformation and plastic strain distribution in the longitudinal section of the pipe in TGM.

Decreasing $q_{\max }^{\prime \prime}$ and increasing $R_{e}$ result in sufficient heating of almost all the thickness. So, the heated area undergoes compressive plastic strain through the thickness and is shortened under the pressure of the surrounding zone. In this heating condition, shortening mechanism occurs which bear dramatic bending of the pipe. The larger the heating area, the larger the plastic area would be, and the shorter the heating area would be created. In Figure $9, \delta$ was maximized by increasing $R_{e}$ and decreasing $q_{\max }^{\prime \prime}$ (relative to the TGM mechanism).

If the thickness of the pipe is small compared to the heating radius, it has low strength against the local buckling. So the heated area was uniformly heated and exposed to local buckling. Figure 10c illustrates this mechanism in a typical pipe. However, this phenomenon did not occur in this study because the pipe was thick. Thus, in the studied pipe, by an excessive increase in the heating radius, the heat was spread throughout the section and reduced the bending resistance. Therefore, the heated zone was exposed to lower pressure from surrounding zone and the compressive plastic strain, and the final bending of the pipe was reduced. As can be seen in Figure 9, excessive increase in $R_{e}$ and decrease in $q_{\max }^{\prime \prime}$ led to the reduction of $\delta$.

The distribution of the longitudinal plastic strain along the pipe length (average of the inner and outer edge values) in low, optimal, and high effective radii $(25,65$, and $100 \mathrm{~mm}$, respectively) at the end of the heating period is shown in Figure 11. The maximum heat intensity is $0.5 \mathrm{~W} / \mathrm{mm}^{2}$ (the optimal value). As can be seen, the amount and extent of the plastic strain in the effective radius of $65 \mathrm{~mm}$ are greater than that for the other radii. Thus, further bending was created in the pipe after cooling.

The residual equivalent plastic strains (after cooling) for the three cases are shown in Figure 12. As can be seen, further and wider residual plastic strain was created in the optimal case.

\subsection{Effect of pipe thickness on the results}

To study the effect of pipe thickness on the optimal values of $R_{e}$ and $q_{\max }^{\prime \prime}$, the analyses were repeated for a pipe with a constant diameter and thicknesses of 3 and $12.7 \mathrm{~mm}$ (based on the available pipe thicknesses in the market). Figures 13 and 14 illustrate the results for the thicknesses of 3 and 


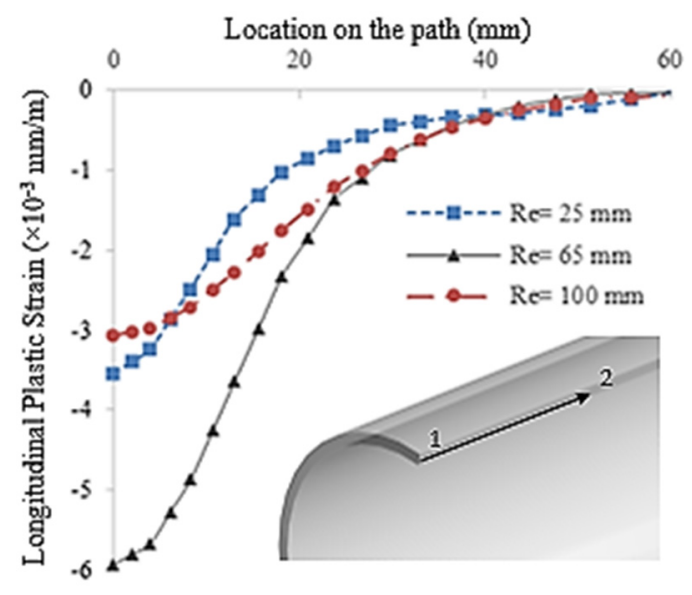

Fig. 11. Longitudinal plastic strain distribution along the longitudinal path at the end of heating time.
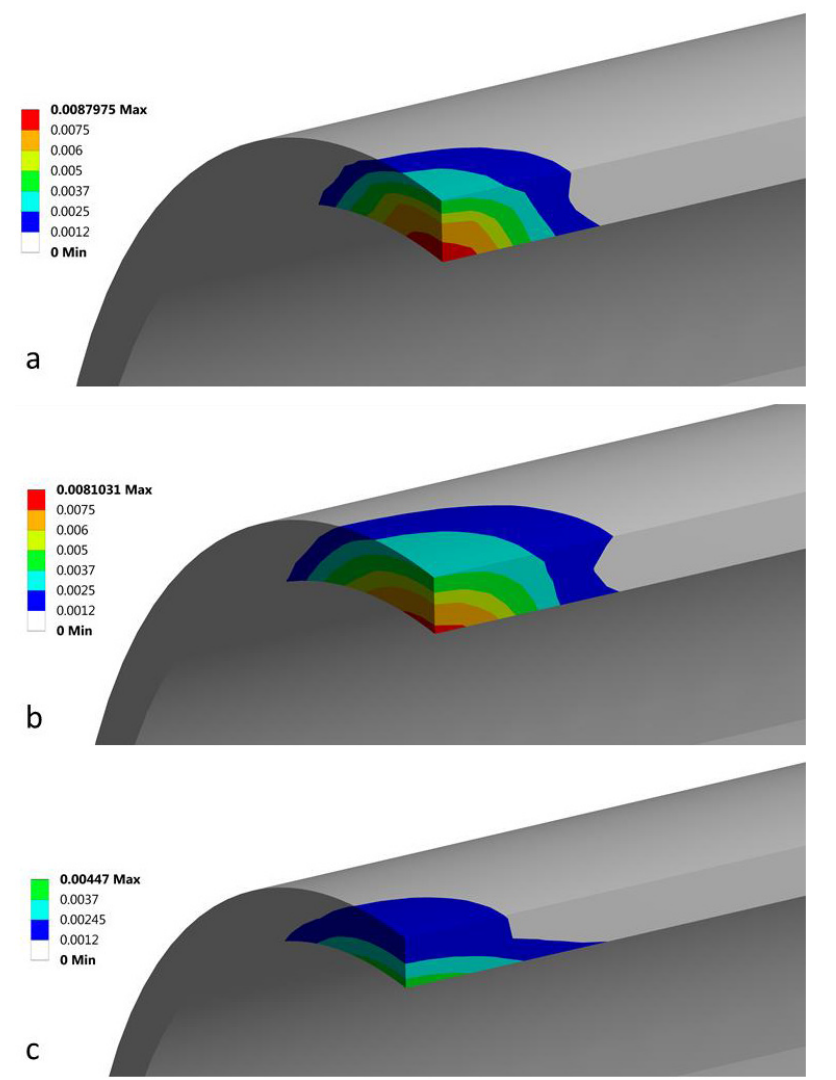

Fig. 12. Residual plastic strain at different effective radii (a) $R_{e}=25 \mathrm{~mm}$, (b) $R_{e}=65 \mathrm{~mm}$, (c) $R_{e}=100 \mathrm{~mm}$.

$12.7 \mathrm{~mm}$, respectively. As can be seen, the overall behavior of the graphs is similar to the pipe with a thickness of $8.18 \mathrm{~mm}$. The distribution of the plastic strain created at the pipe section confirms that the pipe deformation mechanisms were the same in this range of thicknesses.

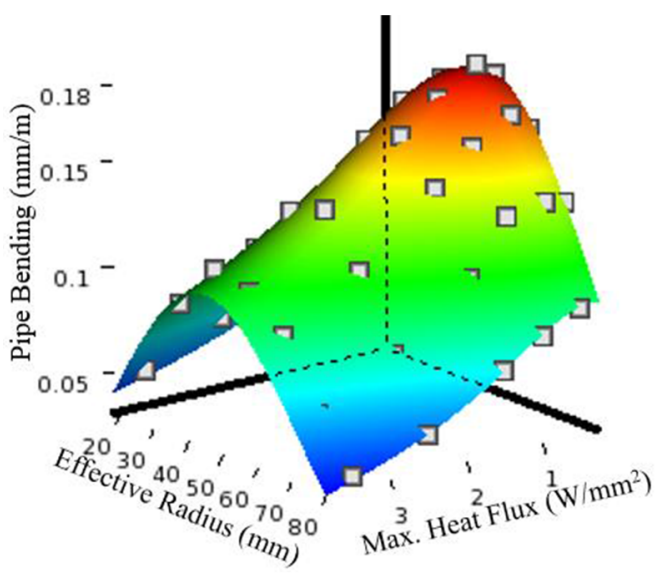

Fig. 13. Variation of displacement vs. effective radius $R_{e}$ and maximum heat flux $q_{\max }^{\prime \prime}$ for the pipe with $3 \mathrm{~mm}$ thickness.

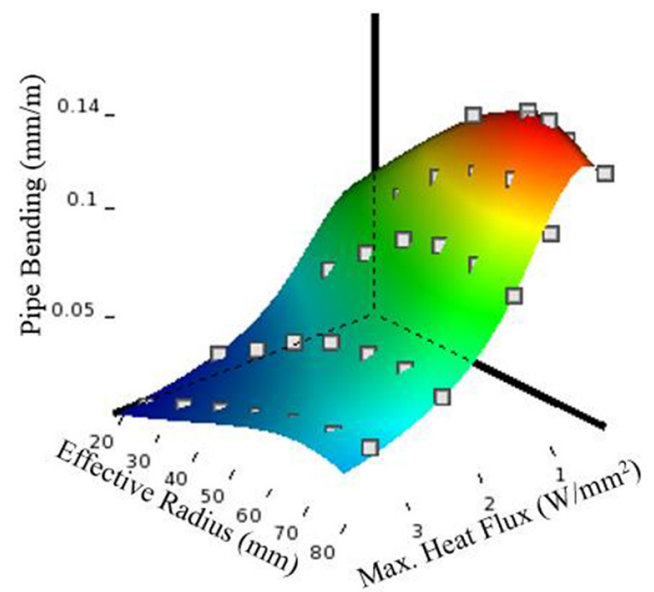

Fig. 14. Variation of displacement vs. effective radius $R_{e}$ and maximum heat flux $q_{\max }^{\prime \prime}$ for the pipe with $12.7 \mathrm{~mm}$ thickness.

Table 1 gives the optimal values of $\delta, R_{e}$, and $q_{\max }^{\prime \prime}$ for pipes with different thicknesses. The smaller the thickness, the more uniform the temperature would be, increasing the local shortening. Increasing the thickness, the heat distribution was not uniform and the plastic strain decreased which resulted in a slight reduction in bending. It should be noted that, although the maximum heat flux intensity did not change significantly with changing the pipe thickness, the heating time varied to reach the maximum temperature.

\subsection{Combination of spot heats}

To increase the pipe bending, it is possible to repeat the heating at a different zone (after cooling the pipe in the previous heating). The repetition of the Spot Heating at a zone with the same heating radius was considered ineffective in pipe bending and even offered a negative 
Table 1. Optimal pipe bending, effective radius, and maximum heat flux in the pipe with different thicknesses.

\begin{tabular}{llll}
\hline $\begin{array}{l}\text { Pipe } \\
\text { thickness } \\
(\mathrm{mm})\end{array}$ & $\begin{array}{l}\text { Optimal heat } \\
\text { flux intensity } \\
\left(\mathrm{W} / \mathrm{mm}^{2}\right)\end{array}$ & $\begin{array}{l}\text { Optimal } \\
\text { effective radius } \\
(\mathrm{mm})\end{array}$ & $\begin{array}{l}\text { Bending } \\
(\mathrm{mm} / \mathrm{m})\end{array}$ \\
\hline 3 & 0.532 & 48 & 0.182 \\
8.18 & 0.528 & 65 & 0.149 \\
12.7 & 0.525 & 66 & 0.140 \\
\hline
\end{tabular}

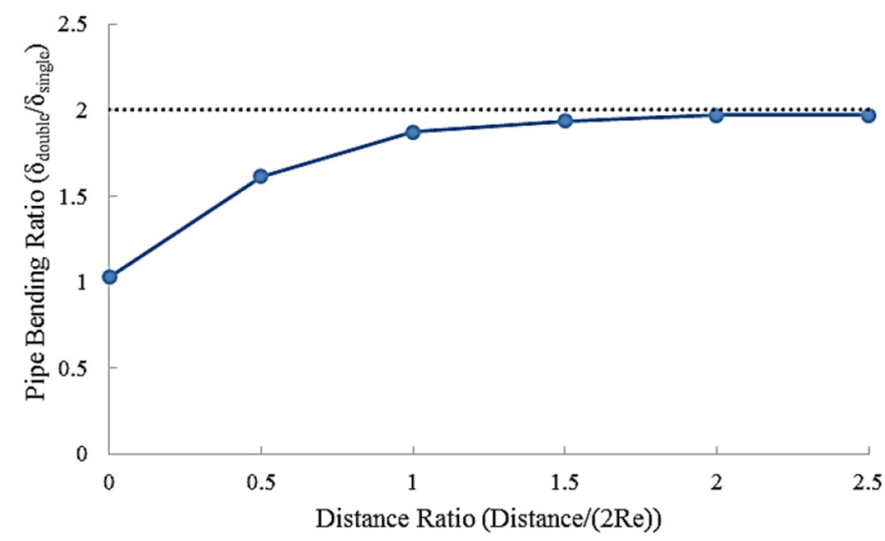

Fig. 15. Effect of distance between two hot spots on the pipe bending ratio.

effect on heating with the smaller radius [5]. Also, the minimum distance for the repetition of heating in the adjacent points (along with the pipe length) should be at least twice the effective diameter of the heating zone. These conclusions were made based on the limited tests performed by Gatto et al. [5] in small effective radii and needed to be further explored. So, the effect of the distance between two heating zones (along the pipe axis) with optimal parameters was investigated.

Figure 15 shows the changes of $\bar{d}$ (ratio of the distance between two heating zones to $2 R_{e}$ ) vs. $\delta_{\text {double }} / \delta_{\text {single }}$ (ratio of the pipe bending in the double-zone heating to the maximum pipe bending in single-zone heating). In the case of two coincident zones $(\bar{d}=0)$, repeating the spot heats was ineffective. But in the case of tangent zones $(\bar{d}=1)$, the repetition of heating led to an increase in the bending by $70 \%$. Moreover, by increasing $\bar{d}$ to 1.5 , the spot heats acted almost independently. Thus, for increasing the pipe bending, it is possible to repeat the spot heats at $\bar{d}=1.5$.

\section{Experimental study}

To evaluate the results of the simulation by FE method, the heating test was performed on a seamless pipe with an inside diameter of 203.2, a thickness of $8.18 \mathrm{~mm}$, and SA-106 material (same dimensions and material used in simulation).

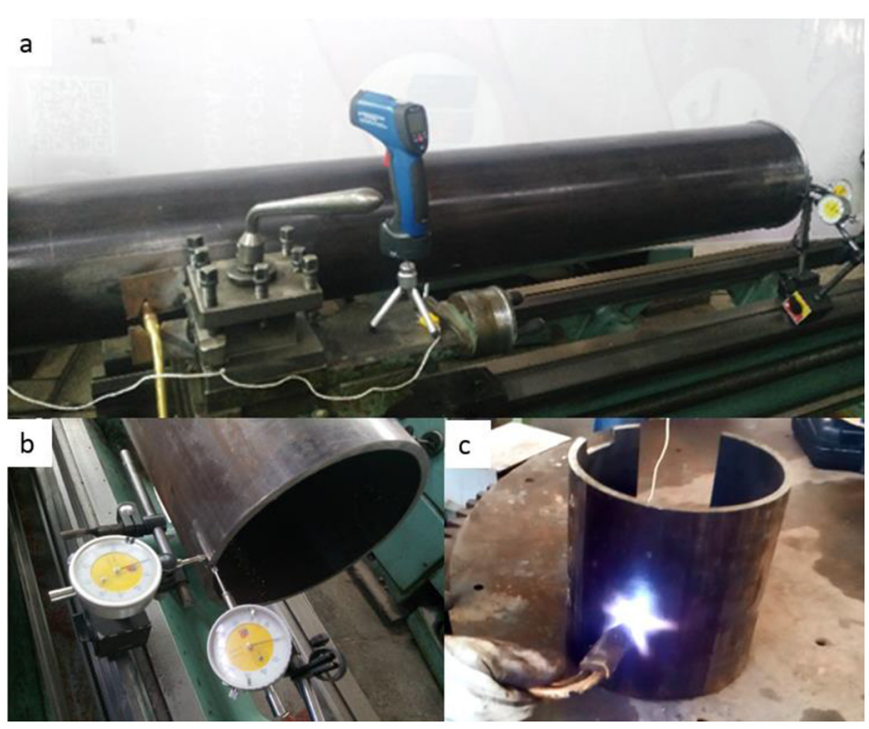

Fig. 16. Pipe Spot Heating test (a) main test, (b) indicators, half-pipe calibration test.

To measure the displacements, the pipe was horizontally fixed to the lathe and two indicators were used with a precision of $0.01 \mathrm{~mm}$ to record the displacement (Fig. 16).

For heating, Oxy-Acetylene torch (Zinser ${ }^{\mathrm{TM}}-$ numbers 3 and 5) were used. Different studies (including $[3-5,10])$ have recommended acetylene or propane gas for Line Heating and Spot Heating (because of high flame temperature). The torch distance from the surface was $20 \mathrm{~mm}$. Determination of heat input is one of the problems of applying a torch. In the present study, an infrared thermo-vision camera was used to determine the surface temperature distribution. Although the surface emissivity coefficient generally has a significant effect on the thermal results obtained from the camera, the emissivity factor was approximately one in the studied pipe due to the surface darkness. A Marmonix ${ }^{\mathrm{TM}}$ thermometer with laser and contact ( $k$ type) probe was used to determine the emissivity coefficient and also to record the surface temperature changes. The temperature of a given point was measured by the contact probe, and the emissivity coefficient was adjusted so that the temperature read by a laser probe was equal to it.

The infrared radiation of the flame yielded errors in measuring the surface temperature using the laser thermometer while it would be more accurate to measure the internal surface of the pipe. Thus, a half-pipe was cut from the same pipe, the torch was placed in front of its outer surface, and the temperature of the inner face was measured by the laser probe. After recording the temperatures over time, the thermal flux in the FEM was changed in such a way that the same temperature variations were obtained. On the contrary, the intensity of the inlet gas and the torch distance can be changed such that the temperature changes over time are similar to the FEM analysis. After calibration of the flame heat flux with halfpipe, the main test was performed with the torch in the 


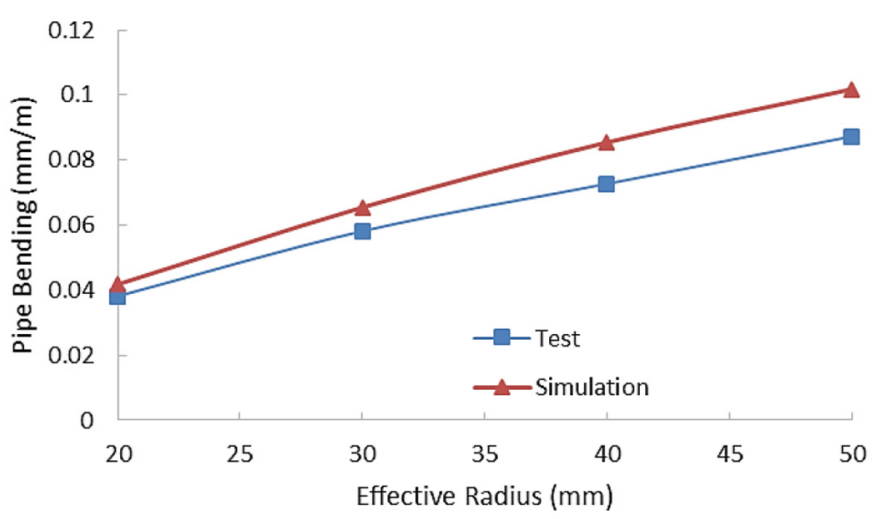

Fig. 17. Comparison of normalized transverse displacement between test and simulation $\left(q_{\max }^{\prime \prime}=1.5 \mathrm{~W} / \mathrm{mm}^{2}\right)$.

absence of any change in the gas intake or the distance from the surface. To have sufficient distance from the lathe fixing jaw, the heating was carried out $300 \mathrm{~mm}$ away from the pipe fixed end. Using different torches and gas intake variations, the flame parameters were adjusted so that the maximum heat flux intensity became $1.5 \mathrm{~W} / \mathrm{mm}^{2}$ at the radii of $20,30,40$, and $50 \mathrm{~mm}$. The pipe bending per unit length of the pipe is shown in Figure 17.

Results show an error range of $9.5 \%-13.7 \%$. The amount of error increased by enlarging effective radius and deviation from the symmetric Gaussian distribution (in the longitudinal and circumferential directions).

\section{Conclusion}

CFD analysis of flame flow was conducted to determine the heat flux distribution over the pipe surface. Then the heating process was simulated to investigate the pipe deformation mechanism and to increase bending in thickwalled steel pipes. The simulation results revealed that as the effective radius increased and the maximum heat flux intensity decreased, the deformation of the pipe increased at first due to the propagation of the plastic zone along the thickness and since the deformation mechanism changed from the thermal gradient to the shortening. By further increasing the effective radius, the heat propagated along the pipe cross-section and caused a decrease in the pipe deformation. The maximum bending of $0.149 \mathrm{~mm} / \mathrm{m}$ was achieved by heating at an effective radius of $65 \mathrm{~mm}$ and a maximum heat flux intensity of $0.528 \mathrm{~W} / \mathrm{mm}^{2}$, which was more than 3 times the bending provided by conventional heating methods $[5,6]$. Although the maximum bending was about 0.15 of that for the Holt method, it can be achieved by combining the Spot Heating at several points, without the complications emerged by controlling the speed and path of the torches. The obtained results showed that the appropriate distance for combination of spot heats should be 1.5 times the heating effective diameter.

Additional studies showed that decreasing and increasing the thickness of the pipe from 8.18 to 3 and $12.7 \mathrm{~mm}$ led to $16 \%$ increase and $6 \%$ decrease, respectively, in the maximum bending of the pipe.

\section{References}

[1] R. Van Gestel, S. Mattheij, Rotor repairs, presented at ASME 1994 International Gas Turbine and Aeroengine Congress and Exposition, The American Society of Mechanical Engineers, The Hague, Netherlands, 13-16 June 1994, pp. 1-9

[2] E. Poursaeidi, M.K. Yazdi, Application of the hot spotting method for the straightening of a large turbine rotor, Int. J. Eng. Trans. A Basics 31 (2017) 110-119

[3] D.M. Richard Avent, Heat Straightening of Damaged Steel Bridge: A Technical Guide and Manual of Practice, US Department of Transportation, Federal Highway Administration, Washington, DC, 1998

[4] A. Varma, Y. Sohn, Effects of realistic heat straightening repair on the properties and serviceability of damaged steel bridges, Publication FHWA/IN/JTRP-2013/03, Joint Transportation Research Program, Indiana Department of Transportation and Purdue University, 2013

[5] F.B. Gatto, D. Mortvedt, C. Smith, J. McMillin, M.W. Baker, Practical Guide for Flame Bending of Pipe, Puget Sound Naval Shipyard, Bremerton, WA, 1991

[6] S. Nelson, J. Dwight, D. Heagy, D. Mortvedt, B. Houghteling, F. Gatto, D. Coglizer, Flame Bending of Pipe for Alignment Control Panel SP-7 Project Report (The National Shipbuilding Program), Puget Sound Naval Shipyard, Bremerton, WA, 1990

[7] X. Wang, J. Wang, W. Xu, D. Guo, Scanning path planning for laser bending of straight tube into curve tube, Opt. Laser Technol. 56 (2014) 43-51

[8] H. Shen, F. Vollertsen, Modelling of laser forming - an review, Comput. Mater. Sci. 46 (2009) 834-840

[9] Y. He, L. Heng, Z. Zhang, Z. Mei, L. Jing, L. Guangjun, Advances and trends on tube bending forming technologies, Chin. J. Aeronaut. 25 (2012) 1-12

[10] H.B. Clausen, Plate Forming by Line Heating, Technical University of Denmark, Kongens Lyngby, 2000

[11] H.B. Clausen, Three Dimensional Numerical Simulation of Plate Forming by Line Heating, ICCAS, Cambridge, MA, 1999

[12] V. Adan, R. Sherif, S. Hisashi, M. Hidekazu, Influential factors affecting inherent deformation during plate forming by line heating (report 1), Trans. JWRI 36 (2007) 57-64

[13] E. Poursaeidi, Y. Kamalzadeh, Causes of rotor distortions and applicable common straightening methods for turbine rotors and shafts, World Acad. Sci. Techno. 55 (2011) $213-218$

[14] R. Hashemi, I. Jalili, M. Abdolmohammadi, Experimental test and finite element analysis of line heating method for forming of ship hull steel plates, Modares Mech. Eng. 14 (2015) 9-16

[15] A. Shahidi, M.M. Nekahi, A. Assempour, Investigation on line heating technique with cooling and determination of the heat paths by strain-based method, Modares Mech. Eng. 16 (2016) 293-304

[16] P. Biswas, N.R. Mandal, O.P. Sha, Thermo-mechanical and experimental analysis of double pass line heating, Mar. Sci. Appl. 10 (2011) 190-198

[17] J.H. Woo, J.G. Shin, Analysis of heat transfer between the torch and the plate for the application of line heating, J. Manuf. Sci. Eng. 125 (2003) 794-800

[18] ANSYS Help System, Swanson Analysis Systems, Houston, PA, 2017 
[19] Y. Shi, Z. Yao, H. Shen, J. Hu, Research on the mechanisms of laser forming for the metal plate, Int. J. Mach. Tool. Manuf. 46 (2006) 1689-1697

[20] W. Li, Y.L. Yao, Laser bending of tubes: mechanism, analysis, and prediction, J. Manuf. Sci. Eng. 123 (2001) 674
[21] S.E. Chavoshi, S.E. Mousavi Torshizi, V. Badali, Deformation mechanism analysis in pipe straightening with spot heating method, presented at 26th Annual International Conference on Mechanical Engineering - ISME 2018, Semnan, Iran, 2018, pp. 431-438

Cite this article as: S.E. Chavoshi, S.E. Moussavi Torshizi, Bending improvement in Spot Heating of pipes in comparison with Line Heating method, Mechanics \& Industry 20, 405 (2019) 